\title{
Rubella-specific IgM detection using Sephacryl S-300 gel filtration
}

\author{
P MORGAN-CAPNER, E DAVIES, AND JR PATTISON \\ From the Departments of Microbiology and Immunology, King's College Hospital Medical School, \\ Denmark Hill, London SE5 8RX, UK
}

SUMMARY Sephacryl S-300 has been compared with Sephadex G-200 in the fractionation of sera for the detection of rubella-specific IgM. No difference in sensitivity was found with sera that had low titres of rubella-specific IgM. Sephadex G-200 was apparently more sensitive with some sera which had high titres of rubella-specific IgM due to the contribution of rubella-specific IgA. Sephacryl S-300 offers considerable advantages in ease and speed of preparation, and as it is a more rigid gel higher flow rates can be obtained. Sera may be fractionated in 2.5 hours on Sephacryl S-300 compared with 7-10 hours on Sephadex G-200.

The investigation of cases and contacts of rubella requires laboratories to be able to detect rubellaspecific IgM antibody. ${ }^{12}$ The methods most widely used to separate serum immunoglobulins are gel filtration and density gradient centrifugation, rubella-specific IgM being detected by haemagglutination-inhibition (HI) tests on the fractions obtained. Currently, the gel of choice in the detection of rubella-specific IgM is Sephadex G-200. More rigid gels could offer considerable advantages in ease of handling and in the time required to fractionate sera since higher flow rates can be used. However, such gels as have been evaluated previously have been found to be unsatisfactory. ${ }^{3}$ Sephacryl S-300 is a dextran gel cross-linked with acrylamide and is the most recently introduced rigid gel. It has therefore been evaluated for the detection of rubellaspecific IgM in comparison with Sephadex G-200.

\section{Material and methods}

GEL FILTRATION

Sephadex G-200 and Sephacryl S-300 were both obtained from Pharmacia Ltd, Uppsala, Sweden. They were used as columns $32 \mathrm{~cm}$ long, $5 \cdot 27 \mathrm{~cm}^{2}$ cross-sectional area in chromatographic columns K26/40 (Pharmacia Ltd). The buffer used for both gel filtration procedures was Tris $(0.02 \mathrm{M})$ buffered $0.9 \% \mathrm{NaCl}, \mathrm{pH} 7 \cdot 5$, containing $0.1 \%$ sodium azide, and flow rates were maintained by hydrostatic pressure.

Received for publication 12 March 1980
Sephadex G-200 was rehydrated by suspending in an excess of buffer and heating in a boiling water bath for 5 hours. The column was prepared by adding an approximately $50 \%$ suspension of gel in buffere and packing to the correct length with a hydrostatic pressure of $10 \mathrm{~cm}$ water. Using this hydrostatic pressure, flow rates of $20-30 \mathrm{ml} /$ hour are obtained. After packing, the gel is equilibrated by passing $400 \mathrm{ml}$ of buffer through it at the above flow rate.

Sephacryl S-300 is supplied preswollen. A $50 \%$ suspension of the gel in Tris buffer is poured into the column, and the gel bed is packed to the required length with a hydrostatic pressure of $150 \mathrm{~cm}$ of water. This hydrostatic pressure gave a flow rate of 80 $\mathrm{ml} /$ hour. The gel is equilibrated by passing $400 \mathrm{ml}$ of buffer through it at the above flow rate.

FRACTIONATION OF SERA

Non-specific inhibitors of rubella virus haemagglutination were removed by prior treatment of the serum with $\mathrm{MnCl}_{2} /$ heparin mixture. ${ }^{4}$ An aliquot of $0.5 \mathrm{ml}$ of this treated serum was layered on to the columns of gel and eluted at the rates stated above for each gel. Fifty 4-ml fractions of the eluant were collected for each fractionation, after which the column was ready for further use. Using the separation conditions stated above, both Sephadex G-200 and Sephacryl S-300 are capable of performing at least $\mathbf{4 0}$ fractionations before repacking is required.

PROTEIN AND IMMUNOGLOBULIN

CONCENTRATIONS

The total protein content eluting from the column 
was continuously monitored as the OD $280 \mathrm{~nm}$ by a Uvicord II spectrophotometer (LKB Produkter AB, Bromma, Sweden).

Levels of immunoglobulins were determined in the undiluted fractions by laser nephelometry using the Behring Laser Nephelometer and laser nephelometer grade antisera to $\alpha, \gamma$, and $\mu$ immunoglobulin chains (Behringwerke, Marburg, West Germany).

\section{RUBELLA-SPECIFIC ANTIBODY TESTS}

Rubella-specific antibody in the immunoglobulincontaining fractions was determined by $\mathrm{HI}$ tests. A microtitre system was used incorporating 4 units of rubella haemagglutinin (Flow Laboratories, Irvine, Scotland), overnight incubation of virus/fraction mixtures, and $0.025 \mathrm{ml}$ of $0.3 \%$ day-old chick erythrocytes as indicator cells.

TREATMENT WITH 2-MERCAPTOETHANOL $0.4 \mathrm{ml}$ aliquots of fractions were incubated with $0.1 \mathrm{ml} 0.5 \mathrm{M} 2$-mercaptoethanol (2ME) for 1 hour at $37^{\circ} \mathrm{C}$. Reaction mixtures were then tested for $\mathrm{HI}$ activity without further treatment.

\section{Results}

Figure 1 compares the elution patterns of a single human serum from columns of Sephadex G-200 and Sephacryl S-300. Serum proteins elute in three distinct peaks from a column of Sephadex G-200. With Sephacryl S-300 the void volume is slightly larger, and protein first elutes one fraction later than with Sephadex G-200. The first protein peak is lower and the second peak tends to merge with the third, which is largely albumin. IgM elutes coincident with the first protein peak with both gels, and the peak IgM is one fraction later with Sephacryl S-300 than with Sephadex G-200. IgG elutes in the second protein peak with both gels, but the peak IgG fraction is 3-4 fractions later with Sephacryl S-300 than with Sephadex G-200. Moreover, with Sephadex G-200, the first IgG containing fraction comes immediately after the last IgM containing fraction, whereas with Sephacryl S-300 there is a gap of two fractions. With both gels IgA elutes in both the first two protein peaks but there is less overlap of $\operatorname{IgA}$ and IgM with Sephacryl S-300.

Figures $2 \mathrm{a}$ and $\mathrm{b}$ show the rubella $\mathrm{HI}$ titres of the immunoglobulin-containing fractions after fractionation of a serum taken 42 days after the onset of a rubella rash. With Sephacryl S-300 there is a clear distinction between the two peaks of HI activity. The smaller first peak consists of rubella-specific IgM antibody since it is eliminated by treatment with 2ME (Fig. 2c). With Sephadex G-200 the two peaks are not so well separated. However, the distribution
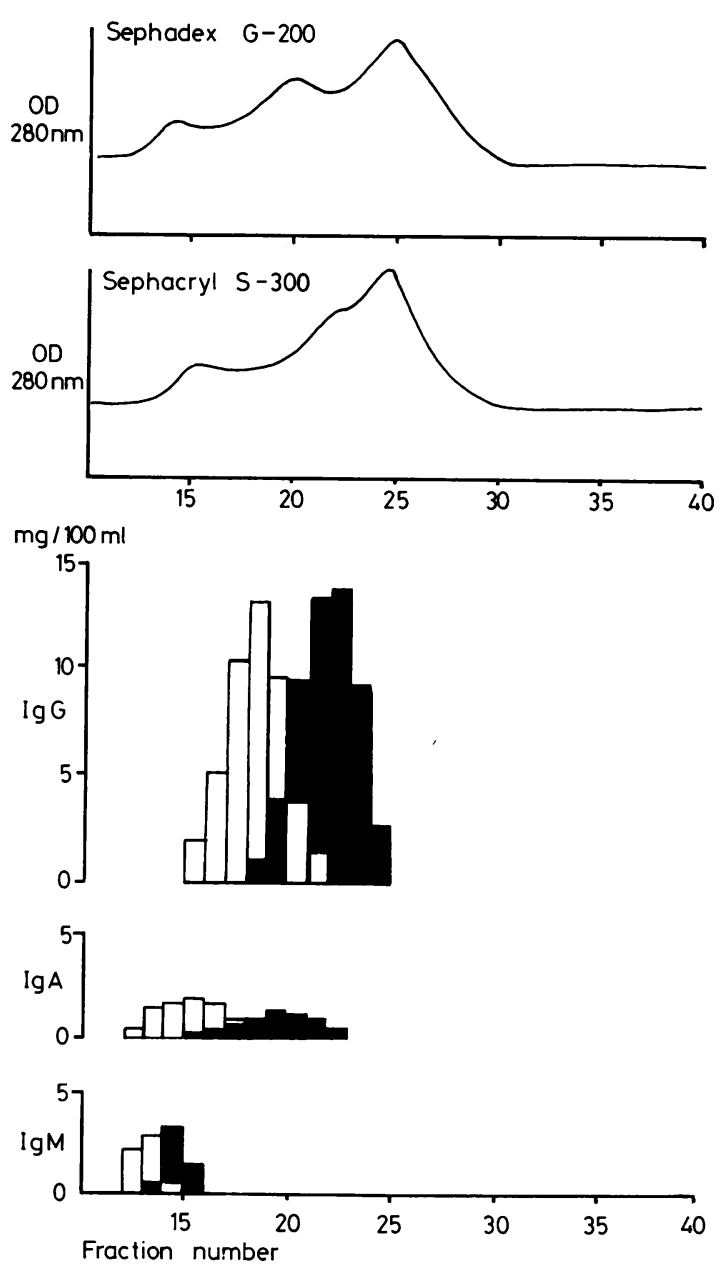

Fig. 1 Elution patterns of serum proteins from Sephadex G-200 and Sephacryl S-300. Hatching denotes the immunoglobulin elution pattern from Sephacryl S-300 as compared with Sephadex G-200 (no hatching).

of $\mathrm{HI}$ activity after $2 \mathrm{ME}$ treatment of all fractions (Fig. 2d) indicates that rubella-specific IgG antibody is not found prior to fraction 15 .

In many early convalescent sera rubella-specific $\operatorname{IgA}$ antibody is present. This fractionates between IgM and IgG with Sephacryl S-300 and blurs the distinction between these two peaks of rubella antibody (Fig. 3). The shaded areas indicate the activity sensitive to $2 \mathrm{ME}$, and this shows that the rubella-specific IgM is confined to the first four immunoglobulin-containing fractions. The remainder of the activity prior to the IgG containing fractions is $2 \mathrm{ME}$ resistant, consistent with its being IgA.

Eleven sera from cases of recent rubella in which rubella $\mathrm{HI}$ activity was present in the $\operatorname{IgM}$ containing 

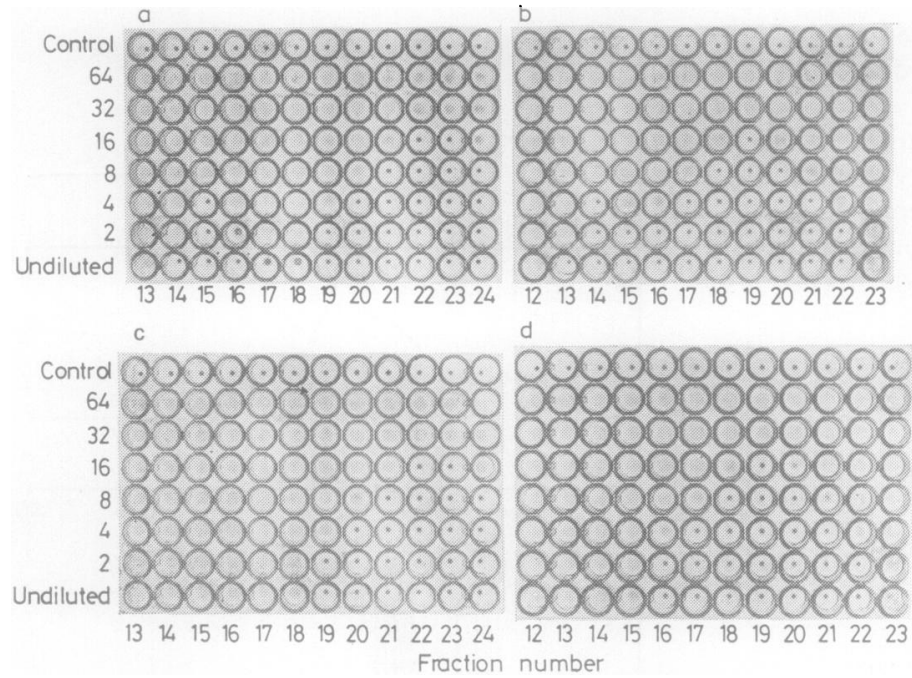

Fig. 2 Rubella HI tests on fractions obtained after fractionation with Sephacryl $S$-300 ( $a$ and $c$ ) and Sephadex G-200 ( $b$ and d) of a serum collected 42 days after $a$ rubella rash. The patterns of reactivity before $(a$ and $b)$ and after ( $c$ and $d)$ treatment of all fractions with $2 M E$ are shown. fractions after fractionation with Sephadex G-200 were evaluated on Sephacryl S-300. The titres obtained in the peak IgM containing fractions are shown in Figure 4. With eight sera there was no significant difference between the two gels in their sensitivity for the detection of rubella-specific IgM. However, in three sera, peak rubella $\mathrm{HI}$ titres of the IgM containing fractions after Sephadex G-200 fractionation were four- to eight-fold higher than those of the IgM containing fractions after Sephacryl S-300 factionation. It should be noted that these three sera gave the three highest titres of $\mathrm{HI}$ activity in the IgM containing fractions after Sephadex G-200 fractionation.

\section{Discussion}

It has been shown previously that Sephadex G-200

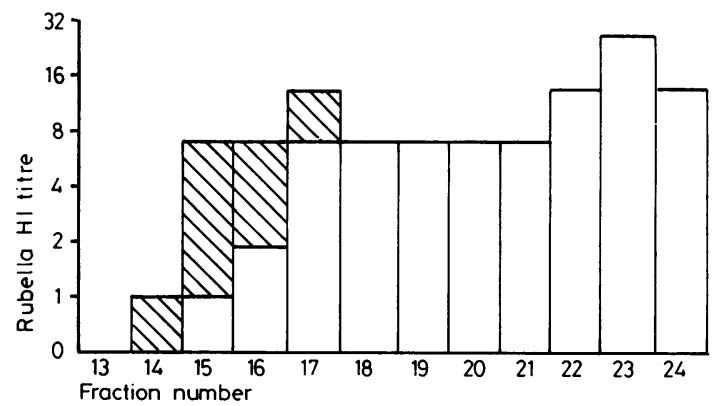

Fig. 3 Rubella HI tests of the immunoglobulin-containing fractions eluted from a column of Sephacryl S-300 for a serum collected eight days after a rubella rash. Hatching denotes sensitivity to $2 M E$.

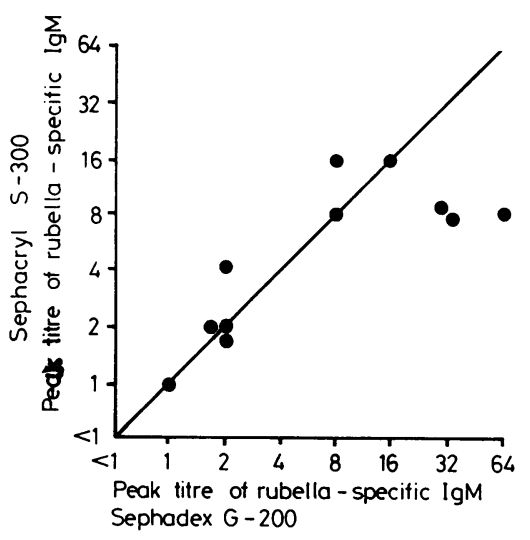

Fig. 4 Comparison of peak HI titres in the IgM containing fractions after fractionation of 11 sera on Sephadex G-200 and Sephacryl S-300.

gel filtration is a sensitive and specific test for rubella-specific IgM. ${ }^{24}$ Fractionation using Sephacryl S-300 offers considerable advantages for the diagnostic laboratory. Sephacryl S-300 is supplied preswollen, and therefore preparation of the column is significantly easier and quicker than for Sephadex G-200, which requires hydration. The principal advantage is the time required to fractionate a serum. With Sephadex G-200 flow rates of 20-30 ml/hour are used, and it therefore requires 7-10 hours to fractionate a serum and regenerate the column for further use. Sephacryl S-300, being a more rigid gel, may be run at a flow rate of $80 \mathrm{ml} /$ hour so that the time taken to fractionate a serum is reduced to 2.5 hours. 
With previously evaluated rigid gels ${ }^{3}$ a loss of sensitivity for the detection of rubella-specific IgM was found. Two factors influence the sensitivity of the gel filtration techniques described: firstly, the number of fractions in which rubella-specific IgM elutes. In this study, using laser nephelometry to detect total IgM, this immunoglobulin elutes in three fractions with each gel. Secondly, sensitivity may be influenced by the elution pattern of rubella specific IgA. With both Sephadex G-200 and Sephacryl $\mathrm{S}-300 \mathrm{IgA}$ is distributed in a broad band between the IgM and IgG peaks. However, the overlap with the IgM containing fractions is considerably greater with Sephadex G-200. The IgA which overlaps with these fractions is dimer IgA. Previous work ${ }^{5}$ has shown that rubella specific antibody of the dimer IgA class in serum is even more transient than the IgM response. Thus with sera taken shortly after the rash dimer IgA contributes more to the first peak of HI activity after Sephadex G-200 fractionation than after Sephacryl S-300 fractionation. This was reflected in the present results with three sera which gave four- to eight-fold higher titres in the IgM containing fractions after Sephadex G-200 fractionation. However, this does not detract from the overall suitability of Sephacryl S-300 for routine diagnostic purposes since there is no difference in sensitivity between the two gels with sera which have smaller amounts of $\mathrm{HI}$ activity in the IgM containing fractions. In these sera, rubella specific IgA contributes little or none of the first peak of HI activity.

A further advantage of Sephacryl S-300 is the greater separation of immunoglobulins that this gel achieves. In using Sephadex G-200, problems of interpretation may arise when sera with high titres of rubella-specific IgG are fractionated. HI activity may 'spill over' into the first protein peak, which is normally associated with IgM. However, because of the greater separation of the IgM and IgG peaks with Sephacryl S-300, the results with such sera are easier to interpret.

Based on the results described and our experience during the past six months, we now regard Sephacryl S-300 as the gel of choice for the detection of rubella-specific IgM.

\section{References}

${ }^{1}$ Field PR, Murphy AM. Specific IgM globulin estimations in the diagnosis of acquired rubella. Med J Aus 1972;2: 1244-8.

2 Pattison JR, Dane DS. The routine serological investigation of cases and contacts of rubella. J Hyg Camb 1975;75: 91-8.

${ }^{3}$ Pattison JR, Jackson C. The use of high speed gel filtration for the detection of rubella-specific IgM. J Immunol Meth 1978;19:269-77.

${ }^{4}$ Pattison JR, Mace JE. The detection of specific IgM antibodies following infection with rubella virus. J Clin Pathol 1975;28:377-82.

${ }^{5}$ Pattison JR, Mace JE. Elution patterns of rubella IgM, IgA, and IgG antibodies from a dextran and an agarose gel. $J$ Clin Pathol 1975;28:670-3.

Requests for reprints to: Dr P Morgan-Capner, Department of Medical Microbiology, King's College Hospital Medical School, Denmark Hill, London SE5 8RX, UK. 